Urologe 2012 $\cdot 51: 615-616$

DOI 10.1007/s00120-012-2858-x

Online publiziert: 27. April 2012

(c) Springer-Verlag 2012
J.W.Thüroff

Urologische Klinik, Universitätsmedizin der Johannes Gutenberg-Universität Mainz, Mainz

\section{Laparoskopische vs. robotische Operationen in der Urologie}

Die angeführten Vorteile der robotisch Laparoskopische Operationen werden ein elementarer Bestandteil des operativen urologischen Armamentariums bleiben, da sie gegenüber den offenen Operationen folgende unumstrittene Vorteile haben: das geringere Accesstrauma, ein geringerer Blutverlust bzw. Transfusionsrate, weniger postoperative Schmerzen bzw. Analgetikaverbrauch und ein günstigeres kosmetisches Ergebnis ( $\bullet$ Abb. 1). Die Fragestellung dieses Heftes befasst sich damit, ob urologische Operationen zukünftig konventionell-laparoskopisch oder robotisch assistiert laparoskopisch durchgeführt werden und welche Argumente für jede einzelne hier diskutierte Operation für die eine oder andere Vorgehensweise sprechen.

Im Allgemeinen werden für robotisch assistierte laparoskopische Operationen folgende Vorteile gegenüber den konventionell-laparoskopischen Techniken angeführt: die dreidimensionale (3D-)Sicht in HDTV, artikulierte Instrumentenbewegungen, ein ausgefilterter Handtremor, die ergonomische Arbeitsposition des Operateurs sowie eine verkürzte Lernkurve (• Abb. 1). Allerdings hat auch im Bereich der konventionell-laparoskopischen Operationstechniken durch Entwicklung von 3D-Optiksystemen, artikulierten Instrumenten und ergonomischen Arbeitsstühlen für den Operateur eine diesbezügliche Weiterentwicklung stattgefunden, die das Manko der konventionellen laparoskopischen Technik möglicherweise reduzieren kann. Trotzdem erscheint der da Vinci ${ }^{\oplus}$-Roboter in seinem derzeitigen Reifegrad mit einer Integration der beschriebenen Faktoren in ein ergonomisch gut funktionierendes Gesamtsystem im Vorteil. assistierten wo es sich um plastisch-rekonstruktive Operationsschritte oder um lang dauernde, komplexe Operationen handelt. Da die allgemeine Vorteile der laparoskopischen Operationstechniken ebenso für konventionell laparoskopische Operationen wie für roboterassistierte Operationen gelten, müssen für den Vergleich zwischen beiden Vorgehensweisen andere Kriterien herhalten, vornehmlich funktionelle und onkologische Ergebnisse, aber auch Faktoren, die eher für den Operateur bzw. die einen Eingriff anbietende Institution als für den Patienten interessant sind wie Operationszeit, Lernkurve, Verfügbarkeit und Kosten des Eingriffs.

Bei der radikalen Prostatektomie ist weltweit eine klare Tendenz der Abkehr von der konventionell-laparoskopischen Technik zur roboterassistierten Operationstechnik erkennbar. Gründe dafür sind ein bereits erreichter hoher Standardisierungsgrad der robotisch assistierten Operationstechnik, berichtete günstige Ergebnisse bei den chirurgischen Absetzungsrändern, der Zeitdauer bis zur postoperativen Wiedererlangung der Kontinenz und den erzielbaren Potenzraten. In den USA liegt der Anteil konventionell laparoskopischer radikaler Prostatektomien derzeit $<1 \%$, ein Fünftel aller Operationen werden noch offen durchgeführt, $80 \%$ robotisch assistiert.

Bei der Nierenbeckenplastik sprechen gute Gründe für die robotisch assistierte Operationstechnik, die aber wegen gleichwertiger Operationsergebnisse, einer relativ kurzen Operationsdauer und eines relativ geringen Grades der Komplexität der Operation nicht zwingend sind. Die Vorteile der laparoskopischen Operationstechnik bezüglich des kosmetischen Ergebnisses und postoperativer Schmerzen/ Bewegungseinschränkungen treten im

\begin{tabular}{|c|c|c|c|}
\hline & Offen & Laparoskopisch & Robotisch \\
\hline \multirow[b]{2}{*}{ Vorteile } & \multirow{2}{*}{$\begin{array}{c}\text { Referenzstandard } \\
\text { OP -Zeit } \downarrow \\
\text { Chirugische Versatilität } \uparrow \\
\text { Wahl der Radikalität } \uparrow\end{array}$} & \multicolumn{2}{|c|}{$\begin{array}{c}\text { Accesstrauma } \downarrow \text { Blutverlust } \downarrow \\
\text { Postoperativer Schmerz } \downarrow \text { Kosmetik } \uparrow\end{array}$} \\
\hline & & Kosten $\downarrow$ & $\begin{array}{c}\text { 3-D Sicht } \\
\text { Artikulierte Instrumente } \\
\text { Handtremor } \downarrow \\
\text { Lernkurve } \downarrow\end{array}$ \\
\hline \multirow[b]{2}{*}{ Nachteile } & \multirow{2}{*}{$\begin{array}{c}\text { Accesstrauma } \uparrow \\
\text { Blutverlust } \uparrow \\
\text { Postoperativer Schmerz } \uparrow \\
\text { Kosmetik } \downarrow\end{array}$} & \multicolumn{2}{|c|}{ OP- Zeit $\uparrow$} \\
\hline & & Lernkurve $\uparrow$ & Kosten $\uparrow$ \\
\hline
\end{tabular}

Abb. $1 \Delta$ Vor- und Nachteile der Operationstechniken 
Vergleich zu offenen Operationen besonders deutlich bei Eingriffen an der Niere zutage, wobei allerdings keine Unterschiede zwischen konventionell laparoskopischer und robotisch assistierter Technik zu erkennen sind.

Bei der Nierenteilresektion bestehen klare Vorteile zugunsten der robotisch assistierten laparoskopischen Technik. Damit lassen sich schwierigste, zentral gelegene Tumoren, teilweise in Zero-Ischämie-Technik organerhaltend operativ entfernen, was konventionell laparoskopisch weltweit nur einigen wenigen spezialisierten Operateuren in gleicher Weise gelingt.

Die Nephrektomie, unabhängig ob als Tumornephrektomie oder als Nephrektomie bei benignen Grundleiden kann zwar robotisch assistiert durchgeführt werden, doch erscheint der erhöhte technische, zeitliche und Kostenaufwand hier gegenüber der konventionell laparoskopischen Nephrektomie kaum gerechtfertigt. Die Nephrektomie wird Domäne konventionell laparoskopischer Operationstechnik bleiben.

Bei der radikalen Zystektomie hat die laparoskopische Operationstechnik gegenüber der offenen Operation zwar Vorteile wie den geringeren Blutverlust, allerdings werden bei anschließender offen chirurgischer Durchführung der Harnableitung andere Vorteile wie das reduzierte Accesstrauma wieder zunichte gemacht. So erscheint es plausibel, dass nur bei komplett intrakorporaler Operation, d. h. der radikalen Zystektomie und Harnableitung als laparoskopische Operation, die Vorteile der laparoskopischen Vorgehensweise dem Patienten gänzlich zugute kommen. Wegen des Fehlens von Langzeitergebnissen bestehen bei der radikalen Zystektomie auch noch Unsicherheiten bezüglich des onkologischen Outcome, so dass die Debatte zwischen offener Operation und laparoskopischer Operationstechnik hier noch nicht ausdiskutiert ist. Allerdings ist die radikale Zystektomie mit intrakorporaler Harnableitung ein gutes Beispiel dafür, dass hier die robotisch assistierte Technik wegen der relativ langen Operationsdauer und der Komplexität des Eingriffs der konventionell laparoskopischen Technik vorzuziehen ist.

Urogynäkologische Deszensusoperationen wie die Sakropexie oder abdomi- nale Netzimplantationen lassen sich sowohl laparoskopisch als auch robotisch assistiert durchführen. Auch hier bestehen bei der Präparation und der Fixation der Netze durch Nähte Vorteile für die robotisch assistierte Technik. Bei vergleichbaren Operationsergebnissen, relativ kurzer Operationsdauer und geringer Komplexität der Operation bestehen hier allerdings keine eindeutigen Vorteile für die robotisch assistierte Operationstechnik.

Die retroperitoneale Lymphadenektomie (RPLA) bei Hodentumorpatienten kann laparoskopisch durchgeführt werden, wobei die bessere Sicht und die artikulierten Instrumente theoretisch einen Vorteil für die robotisch assistierte Operationstechnik bieten würden, insbesondere bei nervenerhaltender RPLA. Allerdings ist heutzutage die Indikation für eine solche Operation selten gegeben, die wesentliche Indikation der RPLA besteht in der Entfernung von Postchemotherapieresiduen, was wegen der Schwierigkeit und teilweise auch Unvorhersehbarkeit (z. B. Gefäßersatz) des Operationsverlaufs am besten mittels offener Operationstechnik durchzuführen ist.

Zusammenfassend ist die robotisch assistierte Operationstechnik auf dem Vormarsch und dies teilweise aus guten Gründen und nicht nur wegen der unsäglichen patientenadressierten Werbung, die einerseits industriegetrieben ist und andererseits auch von den anbietenden Zentren erfolgt. Es sind nicht nur der geringere Blutverlust und weniger postoperative Schmerzen, die die Patienten nach dieser Operationstechnik verlangen lassen, sondern zumindest Gleichwertigkeit zur offenen Operation wenn nicht gar tendenziell bessere funktionelle Ergebnisse sowie eine niedrigere Rate positiver chirurgischer Absetzungsränder. Im Vergleich zwischen offenen und laparoskopischen Operationstechniken sind es die Eingriffe an der Niere, die den deutlichsten Vorteil beim kosmetischen Ergebnis und postoperativen Schmerz zugunsten der laparoskopischen Technik bieten. Unterschiede zwischen konventionell laparoskopischer und robotisch assistierter Vorgehensweise sind hier allerdings nicht erkennbar. Bei den urogynäkologischen Operationen müssen gar ein kosmetischer
Nachteil der verschiedenen Trokarinsertionen gegenüber einem an der Schamhaargrenze liegenden Pfannenstielschnitt konstatiert werden.

Für den Operateur ist ein nicht zu vernachlässigender Vorteil die gute ergonomische Arbeitsposition bei der robotisch assistierten Technik, insbesondere bei lang dauernden und komplexen Eingriffen. Nachteile sind die hohen Kosten mit Anschaffungskosten des da Vinci-Roboters von ca. 1,8-2,2 Mio. EUR, Wartungskosten von ca. $150.000 \mathrm{EUR} / \mathrm{Jahr}$ sowie Verbrauchsmaterialen von ca. 1500 EUR/ Operation, die (je nach Abschreibungsdauer) in ca. 3000-3500 EUR Mehrkosten/Operation resultieren. Damit erscheint eine kostendeckende Durchführung von da Vinci--Operationen im Rahmen des DRG-Systems nicht darstellbar.

Trotzt der "technischen Aufholjagd“" der konventionell laparoskopischen Operationstechnik ist zu erwarten, dass sich die Indikationen für robotisch assistierte Operationen über die hier diskutierten Operationstechniken hinaus weiter ausweiten werden und zunehmend Operationen am Ureter und unteren Harntrakt umfassen werden. Mit einer weiteren Verbreitung der robotisch assistierten Operationstechnik bis hin zur flächendeckenden Vorhaltung ist auch dann zu rechnen, wenn nach Auslaufen der Patentbindung in den Jahren 2013/2014 Konkurrenzsysteme mit hoffentlich niedrigerem Kostenprofil zur Verfügung stehen werden.

\section{Aus Mainz mit kollegialen Grüßen}

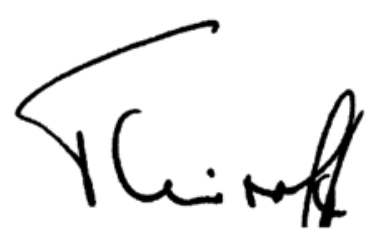

\section{J.W. Thüroff}

\section{Korrespondenzadresse}

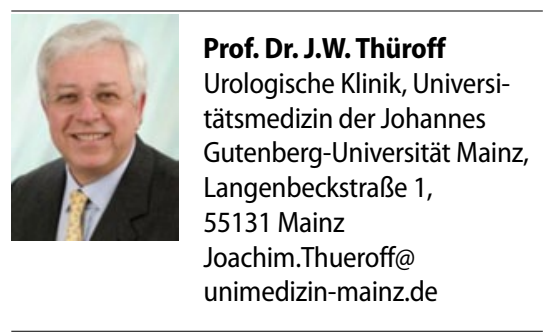

